\title{
Stefano Casarino, Amedeo Alessandro Raschieri (eds.), Ritorno ad Aristotele, Aracne ("Mnemata” 4), Roma 2017, pp. 281, ISBN 978-88-255- 0830-7.
}

"Un classico è un libro che non ha mai finito di dire ciò che ha da dire", suona la sesta proposta di definizione dei classici di Italo Calvino in apertura del saggio Perché leggere $i$ classici. In tal senso Aristotele è paradigmatico e questo volume, il quarto della collana Mnemata, curato da Stefano Casarino e Amedeo A. Raschieri, intende richiamare l'attenzione proprio sulla fortuna e sulla ricezione del filosofo di Stagira dall'epoca bizantina fino ai nostri giorni, passando attraverso il Medioevo islamico e cristiano e il Rinascimento italiano. La miscellanea si presenta divisa in due sezioni: la prima (Ricerca, pp. 31-156) è dedicata a studi di taglio scientifico, la seconda (Didattica, pp. 159-278) è pensata piuttosto per la divulgazione. Dai contributi dell'una e dell'altra emerge chiaramente che con i testi di Aristotele si sono misurati epoche e ambienti culturali diversi e che il suo pensiero, anche qualora mistificato o polemicamente frainteso, è stato capace di ispirare e animare riflessioni e dibattiti dall'antichità fino all'età moderna. Ritorno ad Aristotele significa quindi rintracciare la presenza aristotelica non solo in discipline diverse, secondo quello spirito enciclopedico che animava il filosofo antico e che ha ispirato oggi il progetto stesso di questa miscellanea, ma anche in testi, autori e dottrine non dichiaratamente legate alla tradizione peripatetica.

La sezione più spiccatamente scientifica affronta la ricezione di Aristotele tanto sul piano letterario e filosofico, quanto su quello dell'agire pratico. Il contributo di Lia Raffaella Cresci, Coerenza e incoerenza dell'ñoos nella Poetica. Fortuna e applicabilità di un criterio di valutazione (pp. 31-48), assume come punto di partenza le prescrizioni di Poet. 15, 1454 a 26-32 relative agli elementi a cui il poeta deve mirare nella costruzione dei caratteri tragici,

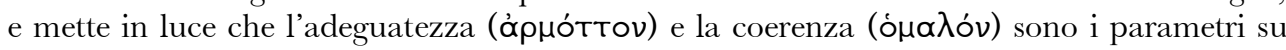
cui molti secoli dopo si fonda l'analisi stilistica di Michele Psello in due opuscoli dedicati al confronto rispettivamente tra Euripide e Giorgio di Pisidia e tra i romanzi di Achille Tazio e di Eliodoro. In particolare, in un genere post-aristotelico quale il romanzo, la coerenza dell' $\hat{n}_{0}$ os, talvolta spinta all'immobilismo, è ciò che compensa e pone rimedio alle peripezie dell'intreccio.

Il lettore si imbatte poi nel noto proverbio una rondine non fa primavera, che rappresenta un'eredità aristotelica: primo tra gli studiosi moderni a dedicare uno studio approfondito a questo detto sentenzioso, Michele Curnis, La rondine di Aristotele (EN 1.7.1098a 18 s.). Un celebre proverbio sulla felicità (pp. 49-89) ne indaga il significato e l'origine e mostra che, se Aristotele fu il primo a servirsene, non per ciò stesso egli ne fu anche l'autore. Con ogni probabilità il filosofo si limitò a riutilizzare un proverbio popolare, adattandolo al proprio discorso: sua sembra la postilla che integra all'idea del manifestarsi episodico della rondine, immagine simbolica dell'esercizio della virtù, la dimensione temporale («una rondine non fa primavera, né la fa un solo giorno», secondo la traduzione di A. Fermani, Aristotele, Le tre Etiche, Milano 2008 citata da Curnis). Oltre ad ampliare l'orizzonte di ricerca dal particolare al generale e a occuparsi della classificazione dei

ПНГН/FONS 3 (2018), 135-138

ISSN 2445-2297 www.uc3m.es/pege
Reseñas bibliográficas (MANUELA CALLIPO) DOI https://doi.org/10.20318/fons.2019.4582 
proverbi nell'analisi retorica aristotelica, questo interessante contributo affianca all'analisi del proverbio da un punto di vista testuale, stilistico e retorico lo studio del contesto in cui esso è

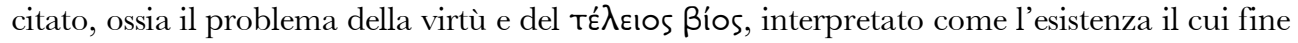
preciso è la felicità. Una sola rondine non basta perché sia davvero arrivata la primavera, ma solo con un tempo adeguato, con la venuta di altre rondini, si avrà la primavera piena e compiuta. Applicata all'esercizio della virtù, che può condurre alla felicità solo se costante, l'immagine della rondine e dell'unico giorno non solo invita alla cautela di fronte a manifestazioni episodiche, ma implica anche che il conseguimento di una realtà piena e compiuta è possibile solo grazie allo scorrere del tempo. Del resto, come leggiamo in Phys. 4.10, 217b-218a, per Aristotele il tempo non può consistere di istanti, ma è un costante divenire, è continuità e movimento. Un solo istante, un solo giorno, il manifestarsi episodico di una sola rondine non sono già più: solo il susseguirsi di giorni e di rondini, solo il perpetuarsi dello stesso fenomeno può portare al compiersi della primavera, e fuor di metafora alla vita felice.

Seguono tre lavori dedicati alla fortuna dei testi aristotelici nel Medioevo e nel Rinascimento. Sergio Carletto, Da Aristotele all'averroismo parigino del XIII sec. (pp. 91108) ripercorre le vicende delle interpretazioni del De anima nel mondo islamico e quindi nell'ambito dell'averroismo parigino, con particolare riferimento al problema del monopsichismo, ossia dell'Intelletto unico e comune a tutti gli esseri umani, trascendente ed eterno; si concentra quindi sulla figura di Sigieri di Brabante, professore della Sorbona che nel 1270 venne condannato dal vescovo di Parigi, Etienne Tempier, per tredici proposte contenute nei suoi scritti e contrarie all'impostazione tomistica. Lo studioso ricostruisce così i dibattiti filosofici e teologici che presuppongono l'esegesi del terzo libro dell'opera aristotelica e della distinzione tra due funzioni dell'intelletto, potenziale e agente, e mette in luce l'evoluzione interna al pensiero di Sigieri, che finì per prendere le distanze da Averroè, in quanto ammise l'unione tra anima intellettiva e corpo e quindi l'individualità del conoscere. All'esegesi medievale e umanistico-rinascimentale sul testo della Politica è dedicato lo studio di Giuliana Besso (pp. 109-136). L'autrice, che alla Politica di Aristotele ha dedicato diverse pubblicazioni (anche in collaborazione con Michele Curnis), traccia un quadro esaustivo della diffusione di questo testo in Europa e mostra come il destino dei libri della Politica si intrecci con il destino delle entità politiche e delle corti europee, che assumono spesso Aristotele a modello e a «fonte di ispirazione per l'agire pratico» (p. 130). Momenti fondamentali della sua ricezione in Europa sono la Francia carolingia, l'opera di traduzione in latino di Guglielmo di Moerbeke (XIII sec.), l'insegnamento di Tommaso d'Aquino, la corte imperiale francese che alla fine del XIV secolo chiese a Nicole Oresme la versione in volgare del testo di Aristotele, e quindi in età umanistica la Firenze di Leonardo Bruni e poi dei Medici, l'Italia della Controriforma con la diffusione dell'opera su più vasta scala anche grazie alla stampa, infine la Spagna e in particolare Saragozza, dove vide la luce per la prima volta nel 1509 una traduzione in volgare, anonima. Se la Politica fu spesso modello e manuale di pedagogia politica, ispirò l'umanesimo civile fiorentino e venne piegata a fornire una base teorica per la costituzione della repubblica di Firenze, misto di democrazia e aristocrazia, G. Besso non ne tralascia la circolazione negli ambienti eruditi, dall'editio princeps di Aldo Manuzio al lavoro di edizione e commento di Pier Vettori, interessati più al testo antico come prodotto letterario che alla sua interpretazione in chiave politica. Di 
particolare interesse non solo per gli specialisti di Aristotele, ma più in generale per la storia della cultura europea è la ricostruzione dell'attività di traduzione dal greco in epoca medievale e soprattutto altomedievale, a conferma dell'ipotesi «che la conoscenza del greco e quindi delle opere aristoteliche in lingua originale - non si sia mai persa del tutto nell'Europa altomedievale, in virtù di quella circolazione di intellettuali e di dotti che ha visto protagoniste soprattutto l'Italia, la Francia e la Germania, per ragioni prioritariamente religiose» (p. 113). Chiude la sezione del volume dedicata alla Ricerca, a coronamento del percorso ideale che dal Medioevo bizantino ha condotto il lettore al Rinascimento italiano, il contributo di Amedeo A. Raschieri, Retorica tra Aristotele e Cicerone nel Rinascimento (pp. 137-156). La fortuna del pensiero retorico aristotelico nell'Umanesimo italiano emerge attraverso l'esame del commento di Nascimbene Nascimbeni al De inventione di Cicerone, pubblicato nel 1563: Nascimbene si serve della teoria aristotelica a sostegno di quella ciceroniana anche contro Quintiliano, che pure ebbe un ruolo di primo piano in ambito retorico e linguistico nell'Umanesimo italiano, e ricorre sia alla Retorica, sia alla Retorica ad Alessandro, da lui considerata genuinamente aristotelica. Aristotele non è tuttavia presenza esclusiva, ma è affiancato da altre fonti greche e latine: Platone, Ermagora, la Rhetorica ad Herennium - che già Nascimbene aveva riconosciuto come non ciceroniana, per quanto vicina all'ambiente di Cicerone - e lo stesso Quintiliano.

Di non minor interesse appaiono i contributi della sezione Didattica, che si devono ad alcuni Docenti di discipline letterarie e scientifiche delle province di Cuneo e Savona. Si tratta di approfondimenti che possono offrire interessanti spunti di riflessione anche a chi insegna nelle scuole superiori di secondo grado. Merito di questo volume è infatti anche quello di rivolgersi a un pubblico non solo accademico e in particolare alle scuole, che rappresentano - o, almeno, dovrebbero rappresentare - il trait d'union tra ricerca specialistica e trasmissione del sapere. Così, Paolo Lamberti (pp. 159-183) si occupa dell'Aristotele biologo e medico, precursore inconsapevole della classificazione delle specie, della teoria della selezione naturale sviluppata da Charles Darwin, dell'idea del metabolismo negli esseri viventi. Sergio Giuliani (pp. 185-199) dedica uno studio al De anima, nel solco delle ricerche relative al problema dell'immortalità dell'anima e del suo rapporto con il corpo, che furono al centro dei dibattiti filosofici nella Parigi del Duecento; così, all'averroismo parigino trattato nella sezione del volume dedicata alla Ricerca si ricollega anche Mauro Lorenzo Somà, L'Averroismo ed i suoi echi letterari nell'opera di Dante e Cavalcanti (pp. 201-215), che offre un quadro ricco e interessante della presenza dell'averroismo nella letteratura medievale italiana. Si procede poi in ambiti più tecnici, con uno sguardo costante al mondo moderno: la filosofia del linguaggio (Giovanni Stefano Lenta, Aristotele e la filosofia del linguaggio ordinario, pp. 217-230), la fisica (Gemma Ghigo, La fisica di Aristotele. Il movimento e il continuo, pp. 231-256), le teorie antiche e moderne relative al regno animale (Luca Maddaloni, Dalla meraviglia aristotelica alle persone non umane, pp. 257-278). La scienza e le тÉXvaı sono naturalmente andate oltre gli insegnamenti aristotelici, ma Aristotele ha avuto il merito di osservare il mondo del sensibile in tutti i suoi aspetti, dal piano umano a

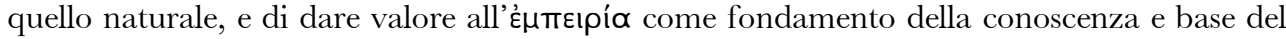
metodo induttivo, per cui «da molte nozioni dell'esperienza si forma un solo giudizio in generale sui casi simili» (Metaph. 981a 6-7). 
Si tratta di un volume ricco e interessante, graficamente curato, utile tanto agli specialisti quanto a un pubblico più ampio. La lettura corre veloce tra filosofia e psicologia, biologia e fisica, filologia e letteratura: grazie alla scansione interna della miscellanea si ha l'impressione di essere accompagnati da un contributo all'altro senza soluzione di continuità, in un percorso ideale in cui ogni argomento presuppone e si lega con armonia con quanto precede.

Manuela Callipo (Liceo Scientifico “Giordano Bruno”, Torino) 